\title{
EFFECTS OF ENVELOPE OVERSHOOT ON THE BUMP OF THE RED GIANT BRANCH LUMINOSITY FUNCTION
}

\author{
M. ALONGI ${ }^{1}$, G. BERTELLI ${ }^{1,2}$, A. BRESSAN ${ }^{3}$, C. CHIOSI $^{1}$ \\ 1Department of Astronomy, University of Padova \\ 2National Council of Research (CNR-GNA) \\ 3Astronomical Observatory of Padova \\ Vicolo dell'Osservatorio 5 \\ I-35122 Padova \\ Italy
}

\begin{abstract}
We show that a certain amount of non-local overshoot at the base of the outer convective envelop of low mass stars, while climbing along the RGB toward central He-ignition, explains the shift of about $0.4 \mathrm{~V}$ mag required to bring the luminosity of the bump predicted in the theoretical RGB luminosity functions into agreement with the observational one.
\end{abstract}

Recent obbservations of the Red Giant Branch (RGB) of Globular Clusters have confirmed the location of a bump in the differential luminosity function. Since the RGB luminosity function maps the hydrogen profile established during central H-burning, the luminosity of the bump identifies the mass-coordinate of the bottom of the homogeneous envelope and, in turn, the depth reached by convection at the time the model was approaching the RGB. Fusi-Pecci et al. (1989) found that the observed luminosity of the bump is fainter than that predicted by current stellar models by about $0.4 M_{v}$. In analyzing the causes of this discrepancy they indicated three main possibilities related to the input physics of the models: the use of old opacities in model calculations, which are lower than the most up-to-date ones for the same metal content (Los Alamos Opacity Library); the treatment of convective mixing; and the choice of the initial helium abundance. They attributed about $\mathbf{0 . 2} \mathrm{mag}$ of the luminosity shift to use of the lower opacities and the remaining 0.2 mag to perhaps a lower helium abundance (say $Y=0.20$ ), while estimating that the amount of overshoot at the base of the convective envelope needed to lower the bump luminosity (about $0.02-0.03 M_{\mathrm{e}}$ ) would be unacceptably high (5 Hp). However, the magnitude shift due to the use of more recent opacities was obtained for a solar metallicity and it is expected to be almost negligible if lower, more appropriate, metallicities are considered. This in turn implies that to explain the magnitude shift one should consider even lower abundances of helium, while, on the other hand, the models of the Sun obtained with the new opacities indicate a higher helium abundance, about 0.27 instead of 0.25 (Vandenberg, 1983).

In this paper we intend to clarify the role played by envelope overshoot. To this aim, we have computed some evolutionary tracks characteristic of the RGB of Globular Clusters, using the new opacities and including overshoot at the base of the convective envelope with different efficiences. The model stars have initial mass of $0.8 M_{0}$, helium abundance of $Y=0.23$, and two extreme metal abundances, i.e. $\mathrm{Z}=0.001$ and $\mathrm{Z}=0.0001$. The efficiency of convective overshoot at the base of the envelope is given by mixing a fraction $\Lambda_{e n}$ of the pressure scale height below the classical boundary, determined by the Schwarzschild criterion. Models are calculated for $\Lambda_{e n}=0.7$ and $\Lambda_{e n}=1.0$. Finally, models with lower initial abundance of helium, $Y=0.20$, are presented for the case without overshoot. The occurrence of overshoot at the base of the convective envelope causes 
additional mixing of about $0.018 M_{\odot}$ or $0.025 M_{\theta}$ for $\Lambda_{e n}=0.7$ and $\Lambda_{e n}=1.0$, respectively, compared to the classical treatment; almost independently of the metal content. The estimate of about five pressure scale heights needed to mix the same amount of matter indicated by Fusi-Pecci et al. (1989) seems to be implausible and likely caused by an evaluation made when the outward migration of the H-burning shell had already stiffened the run of the thermodynamical variables around the chemical discontinuity. To show better how the envelope overshoot affects the luminosity of the bump we plot in Fig. 1 the differential luminosity function, in arbitrary units, for the $\mathrm{Z}=0.001$ and Bell and Gustafsson's (1978) bolometric corrections. The marginal effect of the initial mass function is neglected here. At increasing efficiency of the envelope overshoot, the bump shifts to fainter luminosities and becomes more prominent. This latter fact may be useful to unravel the different explanations. Within the range of $\Lambda_{e n}$ we have investigated, the magnitude shift $\Delta M_{v}$ and the size of the envelope overshoot are correlated by the following relation $\Delta M_{v=} 0.6 \Lambda_{e n}$, which holds good almost independently of the metal content. So, the magnitude shift of about $0.415 M_{\nu}$ in the bump of the RGB luminosity functions suggested by Fusi-Pecci $e t$ al. (1989), can be accounted for by envelope overshoot alone with $\Lambda_{e n}=0.7$. Decreasing the helium content by 0.03 shifts the luminosity of the bump to fainter magnitudes by about $0.15 M_{v}$, as already noted by Fusi-Pecci et al. (1989). However, this way out is not as safe as it may appear, not only because it opposes the current determinations of helium abundance in globular clusters and the estimates from primordial nucleosynthesis calculations, but also because fitting the turn-off of a cluster with a lower helium abundance needs a higher turn-off mass, which compensates for the effect of a lower helium abundance in the bump luminosity as shown by eq. 2 of Fusi-Pecci et al. (1989). An extended discussion on this topic and the implications of envelope overshoot on intermediate mass stars is found in Alongi et al. (1990a, b).

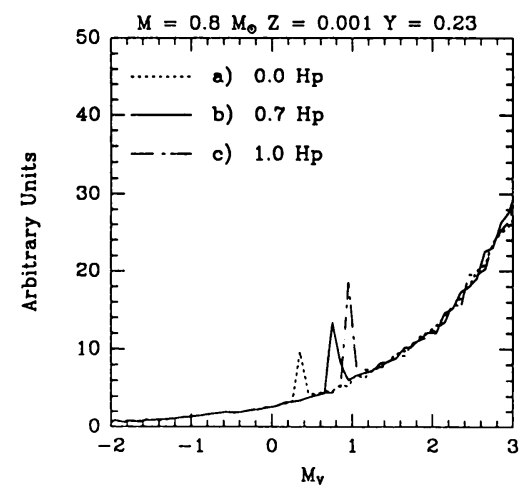

Figure 1. Differential luminosity functions in arbitrary units of the three models with $\mathrm{M}=0.8 M_{\theta}$ and $\mathrm{Z}=0.001, \mathrm{Y}=0.23$ with $\Lambda_{e n}=0, \Lambda_{e n}=0.7$ and $\Lambda_{e n}=1.0 . \Lambda_{e n}$ express the amount of envelope overshoot in units of pressure scale height.

\section{References}

Alongi, M., Bertelli, G., Bressan, A., Chiosi, C. (1990a), Astron. Astrophys. submitted.

Alongi, M., Bertelli, G., Bressan, A., Chiosi, C. (1990b), this volume.

Bell, R.A., Gustafsson, B. (1978), Astron. Astrophys. Suppl. 34, 229.

Fusi-Pecci, F., Ferraro, F.R., Crocker, D.A., Rood, R.T., Buonanno, R. (1989), Astron. Astrophys. submitted.

VandenBerg, D.A. (1983), Astrophys. J. Suppl. Ser. 51, 29. 